\title{
Bosentan treatment in patients with primary pulmonary hypertension receiving nonparenteral prostanoids
}

\author{
M.M. Hoeper, N. Taha, A. Bekjarova, R. Gatzke, E. Spiekerkoetter
}

Bosentan treatment in patients with primary pulmonary hypertension receiving nonparenteral prostanoids. M.M. Hoeper, N. Taha, A. Bekjarova, R. Gatzke, E. Spiekerkoetter. (C) ERS Journals Ltd 2003.

ABSTRACT: Primary pulmonary hypertension (PPH) is a life-threatening disease. Nonparenteral prostanoids, i.e. aerosolised iloprost or oral beraprost sodium show beneficial therapeutic effects but are not sufficiently active in all patients with this devastating disease. The purpose of the present study was to determine whether the endothelin-receptor antagonist bosentan is safe and effective in patients with PPH already receiving nonparenteral prostanoids.

The effect of bosentan as add-on medication was studied in 20 patients with $\mathrm{PPH}$, who received either inhaled iloprost $(n=9)$ or oral beraprost $(n=11)$ for a median period of $16 \pm 13$ months, by means of the 6-min walk test and cardiopulmonary exercise testing.

After 3 months of administration of bosentan in addition to prostanoids, the walking distance in the 6-min walk test increased by $58 \pm 43 \mathrm{~m}$. Cardiopulmonary exercise testing revealed an increase in maximal oxygen consumption from $11.0 \pm 2.3$ to $13.8 \pm 3.6 \mathrm{~mL} \cdot \mathrm{kg}^{-1} \cdot \mathrm{min}^{-1}$ accompanied by significant improvements in anaerobic threshold, oxygen pulse and minute ventilation/carbon dioxide production slope. Peak systolic blood pressure increased from $120 \pm 17$ to $139 \pm 21 \mathrm{mmHg}$. Combination treatment was well tolerated by all patients.

It is concluded that the addition of the endothelin-receptor antagonist bosentan to inhaled iloprost or oral beraprost therapy appears to be safe for patients with primary pulmonary hypertension, resulting in a marked increase in exercise capacity. Therefore, rigorous studies should address whether combination treatment is more effective than either therapeutic intervention alone.

Eur Respir J 2003; 22: 330-334.
Hannover Medical School, Hannover, Germany.

Correspondence: M.M. Hoeper

Dept of Respiratory Medicine

Hannover Medical School

30623 Hannover

Germany

Fax: 495115328536

E-mail: hoeper.marius@mh-hannover.de

Keywords: Cardiopulmonary exercise testing endothelin-receptor antagonists

prostaglandins

pulmonary hypertension

Received: January 232003

Accepted after revision: February 142003
Primary pulmonary hypertension (PPH) is a disease of unknown origin, characterised by progressive obliteration of small- and medium-sized pulmonary arteries [1]. Without appropriate medical treatment, the mean survival of patients suffering from $\mathrm{PPH}$ is $<3$ yrs [2].

Continuous intravenous infusion of prostacyclin (epoprostenol) has been the preferred treatment of PPH for many years [3, 4]. Although it is undisputed that this treatment is highly effective, there are significant risks associated with the permanent central venous access and delivery system required for an uninterrupted supply of epoprostenol [5, 6]. These problems have led to the development of novel prostacyclin derivatives, which can be administered subcutaneously, orally or by means of inhalation. Randomised, placebo-controlled clinical trials have demonstrated the clinical efficacy of subcutaneous treprostinil [7], oral beraprost sodium [8] and inhaled iloprost [9]. Owing to their noninvasive nature, it seems appropriate to use these new substances in the first-line treatment of patients with $\mathrm{PPH}$, reserving intravenous prostaglandins for the most severe cases and treatment failures [10].

Bosentan is the first representative of a new class of drugs, acting as an endothelin-receptor antagonist [11]. Two

For editorial comments see page 193. controlled trials have demonstrated that bosentan improves exercise capacity in patients with PPH and pulmonary arterial hypertension $(\mathrm{PAH})$ associated with systemic sclerosis [12, 13]. Bosentan was the first oral drug approved by the US Food and Drug Administration and their European counterpart the European Agency for the Evaluation of Medicinal Products for treatment of PPH and PAH associated with collagen vascular disease. Consequently, physicians can now choose between several prostaglandins or endothelin-receptor antagonists for the first-line treatment of patients with PPH.

Experiences with continuous intravenous epoprostenol suggests that the long-term prognosis of patients with $\mathrm{PPH}$ is favourable only if they can be stabilised in New York Heart Association Functional Class II with a walking distance of $>380 \mathrm{~m}$ in the 6-min walk test [5]. It is obvious that such ambitious goals may not be reached by all patients receiving nonparenteral prostaglandins or endothelin-receptor antagonists alone. Under these circumstances, a combination regimen of prostanoids and endothelin-receptor antagonists might be the therapy of choice. Simultaneous administration of intravenous epoprostenol and bosentan is being studied in the Bosentan Randomized Trial of Endothelin Receptor Antagonist Therapy (BREATHE)-2 trial, the results of which have not yet been published. No study to date has evaluated the effects of nonparenteral prostanoids combined with bosentan in males, whereas data from animal studies suggest that 
combination treatment is more effective than treatment with prostanoids or bosentan alone [14].

In the present pilot trial, the efficacy and safety of bosentan was studied in patients with PPH already receiving either inhaled iloprost or oral beraprost. Adverse events were carefully recorded and efficacy was determined using the 6-min walk test and cardiopulmonary exercise testing (CPET).

\section{Methods and patients}

\section{Patients}

Patients with PPH were eligible for the present pilot trial if treatment with either inhaled iloprost or oral beraprost had been administered at a stable dose for $\geqslant 3$ months. The clinical efficacy of the treatment was deemed unsatisfactory in any of the following situations: 1) the walking distance in the 6-min walk test did not reach $350 \mathrm{~m}$ with prostanoid treatment; 2) the walking distance in the 6-min walk test reached $>350 \mathrm{~m}$ after initiation of prostanoid treatment but eventually declined by $\geqslant 50 \mathrm{~m}$ from the best individual value on two consecutive measurements; and 3) right heart catheterisation revealed a low cardiac output defined as a cardiac index of $<2.5 \mathrm{~L} \cdot \mathrm{min}^{-1} \cdot \mathrm{m}^{-2}$ at rest despite prostanoid treatment.

All patients were informed about the alternative possibility of continuous prostacyclin infusion, considered an established treatment. They were aware of the fact that bosentan was a novel drug with unexplored long-term safety and efficacy profiles and knew about the lack of experience concerning combination treatment. In addition, the patients were informed about the potential risks of CPET. The institutional review board approved this protocol and all patients gave their written informed consent.

\section{Treatment}

Inhaled iloprost was delivered by either jet or ultrasound nebuliser [15-17]. Beraprost was titrated to the maximum tolerable dose according to the protocol of the Arterial Pulmonary Hypertension and Beraprost European Trial (ALPHABET) [8].

The doses of iloprost and beraprost were kept stable for $\geqslant 3$ months before initiation of combination treatment and were not changed throughout the study. Bosentan was given at $62.5 \mathrm{mg}$ twice daily for 4 weeks followed by $125 \mathrm{mg}$ twice daily unless elevations of liver enzyme concentrations or other medical reasons prohibited dose escalation [13].

\section{Efficacy}

Efficacy was determined by assessing walking distance in the unencouraged 6-min walk test [18] and CPET. All variables were examined prior to initiation of bosentan therapy and for 3 months thereafter. Further 6-min walk tests were performed every 3 months. The CPET protocol was adapted with minor modifications from SUN and co-workers [19, 20]. Each patient underwent physician-supervised progressively increasing work-rate CPET to maximum tolerance on an electromagnetically braked cycle ergometer. After a resting period of $3 \mathrm{~min}$, patients were asked to cycle at 60 revolutions per minute while the work rate was increased by 5-15 W. $\min ^{-1}$ to maximum tolerance. Gas exchange measurements were made continuously (Oxycon Champion; Jaeger, Würzburg, Germany). Cardiac frequency, pulse oximetry, 12-lead electrocardiography and blood pressure results were monitored and recorded.

Minute ventilation $\left(V^{\prime} \mathrm{E}\right)$ at body temperature and ambient pressure, saturated with water vapour, and oxygen consumption $\left(V^{\prime} \mathrm{O}_{2}\right)$ and carbon dioxide production $\left(V^{\prime} \mathrm{CO}_{2}\right)$, both at standard temperature and pressure, dry, were measured breath by breath, interpolated second by second and averaged over 10-s intervals. The anaerobic threshold was determined as described by WASSERMAN et al. [21]. Ventilatory efficacy during exercise was expressed as the ratio between $V^{\prime} \mathrm{E}$ and $V^{\prime} \mathrm{CO}_{2}$ at the anaerobic threshold. Ventilatory efficacy was analysed only in patients without a persistent foramen ovale [20].

In order to avoid acute haemodynamic effects, especially as a result of treatment with iloprost and beraprost, all patients had to observe an interval of $\geqslant 2 \mathrm{~h}$ between the last intake of any medication and the exercise test [22, 23]. Right heart catheter examinations were performed in all patients within the 6 months prior to bosentan therapy (while the patients were already receiving prostanoid treatment) but catheter studies were not used to assess the effects of combination treatment.

\section{Safety}

The first six patients were admitted to Hannover Medical School (Hannover, Germany) for initial treatment with bosentan in combination with prostanoids because of concerns about hypotensive episodes. When no such episodes were observed, all further patients started treatment at home after having been informed about potential side-effects. All patients were advised to measure and record their blood pressures and cardiac frequencies at least three times daily.

Liver enzyme concentrations, bilirubin levels, international normalised ratios (INRs) and haemoglobin levels were measured prior to treatment with bosentan and at 4-weekly intervals. Additional measurements of liver enzymes, bilirubin and INR were scheduled 2 weeks after alteration of bosentan dosage.

\section{Statistical analysis}

Results are presented as mean \pm SD. Results obtained at baseline and after 3 months of treatment with bosentan were compared using a paired t-test. All tests were two-sided. A p-value of $<0.05$ was considered significant.

\section{Results}

Combination treatment was initiated in 21 consecutive PPH patients. In one patient, liver enzyme concentrations had increased 10-fold compared to baseline levels 4 weeks after bosentan therapy had been started. It is worth noting that this patient reported having a bout of heavy alcohol consumption during this time. Bosentan was withdrawn and not reinstituted, causing the patient to be excluded from further analysis. Three months later, liver enzyme concentrations were improved but had not returned to normal levels. The demographic characteristics and baseline parameters of the remaining 20 patients eligible for analysis are shown in table 1 .

Addition of bosentan treatment resulted in subjective improvement in exercise tolerance and reduction in dyspnoea during physical activity in all patients within 1-2 weeks. Prior to bosentan treatment, 17 patients were in World Health Organization (WHO) functional class III and three in class 
Table 1.-Baseline characteristics of primary pulmonary hypertension patients receiving combination treatment

\begin{tabular}{|c|c|}
\hline Patients $\mathrm{n}$ & 20 \\
\hline Females/males $\mathrm{n}$ & $14 / 6$ \\
\hline Age yrs & $46 \pm 10$ \\
\hline NYHAFC III/IV n & $17 / 3$ \\
\hline PFO present/absent $n$ & $8 / 12$ \\
\hline Time since diagnosis months & $23 \pm 18$ \\
\hline Prostanoid treatment duration months & $16 \pm 13$ \\
\hline \multicolumn{2}{|l|}{ Prostanoid dose $\mathrm{e}^{\#} \mu \mathrm{g} \cdot \mathrm{day}^{-1}$} \\
\hline Iloprost (aerosolised) & 30 \\
\hline Beraprost & 240 \\
\hline \multicolumn{2}{|l|}{ Haemodynamics } \\
\hline Pra $\mathrm{mmHg}$ & $6 \pm 5$ \\
\hline Mean $P$ pa $\mathrm{mmHg}$ & $55 \pm 11$ \\
\hline $\mathrm{CI} \mathrm{L} \cdot \mathrm{min}^{-1} \cdot \mathrm{m}^{-2}$ & $1.9 \pm 0$. \\
\hline PVR dyn $\cdot \mathrm{s} \cdot \mathrm{cm}^{-5}$ & $1147 \pm 32$ \\
\hline $\mathrm{SV} \mathrm{mL}$ & $42 \pm 10$ \\
\hline $\mathrm{Pa}, \mathrm{O}_{2}$ torr & $67 \pm 12$ \\
\hline$S_{\mathrm{V}, \mathrm{O}_{2}} \%$ & $59 \pm 7$ \\
\hline
\end{tabular}

Data are presented as mean \pm SD or absolute numbers unless otherwise indicated; NYHAFC: New York Heart Association Functional Class; PFO: persistent foramen ovale; Pra: right atrial pressure; $P$ pa: mean pulmonary arterial pressure; $\mathrm{CI}$ : cardiac index; PVR: pulmonary vascular resistance; $\mathrm{SV}$ : stroke volume; $\mathrm{Pa}, \mathrm{O}_{2}$ : arterial oxygen tension; $\mathrm{S}_{\mathrm{v}, \mathrm{O}_{2}}$ : venous oxygen saturation. "\#: median; ": dose delivered at mouthpiece. 1 dyn $=10^{-5} \mathrm{~N} ; 1$ torr $=0.133 \mathrm{kPa}$.

IV; after 3 months of bosentan treatment, one patient remained in functional class IV (although the walking distance in the 6-min walk test increased from 66 to $132 \mathrm{~m}$ ), 13 patients were in WHO class III and six in class II. This effect was sustained throughout the 3-month observation period and no patient required switching to intravenous prostaglandin treatment. Owing to their clinical improvement, three patients, who were on a waiting list for lung transplantation, asked to be removed from the active list. Consent was given since the 6-min walk tests and CPET confirmed marked improvement in exercise capacity.

\section{Six-minute walk test}

The walking distance in the 6-min walk test increased from $346 \pm 106 \mathrm{~m}$ at baseline to $404 \pm 101 \mathrm{~m} 3$ months after initiation of bosentan treatment $(\mathrm{p}<0.0001)$. At the time of writing, 11 patients had received combination treatment for 6 months. In these patients, the walking distance in the 6-min walk test was $361 \pm 70 \mathrm{~m}$ before bosentan treatment was started, $425 \pm 59 \mathrm{~m}$ after 3 months and $444 \pm 60 \mathrm{~m}$ after 6 months, suggesting further improvement in exercise capacity with longer treatment. Statistical comparisons of the results after 3 and 6 months were not performed because of the small number of patients.

\section{Cardiopulmonary exercise testing}

CPET was completed without adverse event in all patients. The anaerobic threshold was reached in all but one patient and end-exercise respiratory exchange ratios were not significantly different at baseline and follow-up $(1.12 \pm 0.09$ versus $1.14 \pm 0.08 ; \mathrm{p}=0.3$ ), indicating that all patients had exercised to maximal intensity at both baseline and follow-up. As shown in table 2, bosentan treatment resulted in substantial and significant increases in maximum work rate, maximal $V^{\prime} \mathrm{O}_{2}$ $\left(V^{\prime} \mathrm{O}_{2}, \mathrm{max}\right)$, anaerobic threshold, oxygen pulse and peak systolic blood pressure. Individual changes in $V^{\prime} \mathrm{O}_{2}$, max are shown in figure 1 . The ventilatory efficacy was calculated only
Table 2.-Cardiopulmonary exercise testing results at baseline and 3 months after addition of bosentan treatment

\begin{tabular}{|c|c|c|c|}
\hline & Baseline & 3 months & $\mathrm{p}$-value \\
\hline Work rate $\mathrm{W}$ & $43 \pm 20$ & $63 \pm 17$ & $<0.0001$ \\
\hline$f_{\mathrm{c}, \max }$ beats $\cdot \min ^{-1}$ & $133 \pm 16$ & $139 \pm 16$ & 0.03 \\
\hline$V^{\prime} \mathrm{O}_{2}, \max \mathrm{mL} \cdot \mathrm{min}^{-1} \cdot \mathrm{kg}^{-1}$ & $11.0 \pm 2.3$ & $13.8 \pm 3.6$ & $<0.0001$ \\
\hline $\mathrm{AT} \mathrm{mL} \cdot \mathrm{min}^{-1} \cdot \mathrm{kg}^{-1}$ & $10.2 \pm 2.2$ & $11.7 \pm 2.9$ & 0.007 \\
\hline Peak oxygen pulse $\mathrm{mL} \cdot$ beat $^{-1}$ & $5.7 \pm 1.5$ & $6.8 \pm 2.0$ & 0.0004 \\
\hline$V^{\prime} \mathrm{E}, \max \mathrm{L} \cdot \mathrm{min}^{-1}$ & $45.8 \pm 14.5$ & $57.6 \pm 15.0$ & $<0.0001$ \\
\hline$V^{\prime} \mathrm{E} / V^{\prime} \mathrm{CO}_{2}$ at $\mathrm{AT}^{\#}$ & $52.3 \pm 14.9$ & $44.9 \pm 10.3$ & 0.004 \\
\hline Peak SBP mmHg & $120 \pm 17$ & $139 \pm 21$ & $<0.0001$ \\
\hline
\end{tabular}

Data are presented as mean $\pm \mathrm{SD}$. $f_{\mathrm{c}, \text { max: }}$ maximal cardiac frequency; $V^{\prime} \mathrm{O}_{2}$,max: maximal oxygen consumption; AT: anaerobic threshold; $V^{\prime}$ E,max: maximal minute ventilation; $V^{\prime} \mathrm{CO}_{2}$ : carbon dioxide production; $V^{\prime} \mathrm{E} / V^{\prime} \mathrm{CO}_{2}$ : ventilatory efficacy; SBP: systolic blood pressure. ${ }^{\#}$ : determined only in 12 patients without a persistent foramen ovale; ${ }^{\circ}$ : at maximum exercise.

in those 12 patients without a persistent foramen ovale and also showed significant improvement (table 2).

\section{Side-effects, safety and drug interactions}

Combination treatment was well tolerated by all patients. Episodes of neither symptomatic hypotension nor syncope were seen. Elevations of liver enzyme concentrations were observed in two patients. In one patient, aspartate aminotransferase and alanine aminotransferase levels had risen to three times the upper normal limit 4 weeks after initiation of bosentan. This patient received an equal dose of $62.5 \mathrm{mg}$ twice daily for another 4 weeks, after which enzyme levels had returned to normal. Dose escalation to $125 \mathrm{mg}$ twice daily did not result in another increase in liver enzyme concentrations. The second patient has already been mentioned above. Mild leg oedema was observed in 10 patients and was mostly spontaneously reversible without specific treatment. Four patients required temporary increases in their diuretic medication. Although minor decreases in haemoglobin level were observed in one-half of the patients, there was no decline in haemoglobin levels of $>2 \mathrm{~g} \cdot \mathrm{dL}^{-1}$ in any patient. INRs decreased in 17 of 20 patients, requiring increased doses of the oral anticoagulant phenprocoumon (an anticoagulant

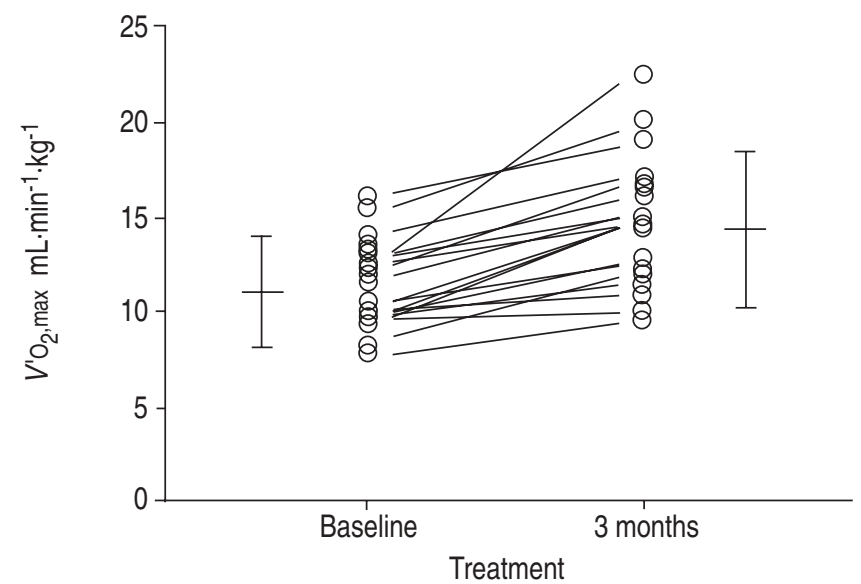

Fig. 1.- Maximal oxygen consumption $\left(V^{\prime} \mathrm{O}_{2}, \max \right)$ on treatment with either aerosolised iloprost or oral beraprost alone (baseline) and 3 months after addition of bosentan treatment with an unchanged dose of prostanoid in 20 patients with primary pulmonary hypertension. Vertical bars represent mean \pm SD. 
used instead of warfarin in Germany and other European countries) in all of these patients.

In 11 patients, follow-up was extended for up to 6 months without any further adverse events.

\section{Discussion}

The results of the present study suggest that addition of the endothelin-receptor antagonist bosentan to nonparenteral prostanoids is highly effective in patients with PPH. The 20 patients who received bosentan in addition to either inhaled iloprost or oral beraprost achieved substantial increases in their walking distances in the 6-min walk test. In addition, CPET revealed highly significant increases in maximum work rate, $V^{\prime} \mathrm{O}_{2}$, max, anaerobic threshold and peak systolic blood pressure during maximal exercise. Furthermore, there was a significant increase in oxygen pulse, indicating an increase in cardiac stroke volume [21]. Together, these data suggest a substantial improvement in cardiac function during exercise. Although catheter studies were not performed, it seems likely that adding bosentan to prostaglandins increases pulmonary blood flow, at least during exercise. This hypothesis is supported by the observed improvement in ventilatory efficacy $\left(V^{\prime} \mathrm{E} / V^{\prime} \mathrm{CO}_{2}\right)$ in patients receiving combination therapy. It is well known that ventilatory efficacy is impaired in patients with PPH, primarily as a result of underperfused ventilated alveoli. Consequently, an improvement in ventilatory efficacy most probably indicates some redistribution of pulmonary perfusion in patients with PPH.

Patients receiving bosentan in the present observational clinical trial were treated at a stable dose of inhaled iloprost or oral beraprost over a period of $16 \pm 13$ months. Although some beneficial effects were initially achieved with both medications in all patients, the individual long-term efficacy of these treatments was insufficient since all patients showed signs of clinical deterioration despite ongoing treatment. The results of the present study suggest that in cases of insufficient efficacy of inhaled iloprost or oral beraprost, a combination of medications with different modes of action may be a reasonable and effective approach. However, further data are still needed to validate this hypothesis.

The medications currently available do not cure PPH. Although the beneficial effects of novel prostaglandins and bosentan on exercise capacity and haemodynamics have been demonstrated in numerous trials, it is not known whether these medications improve long-term survival. However, several studies provide ample evidence that exercise capacity and survival in patients with PPH are closely related. Mічамото et al. [24] have shown that walking distance in the 6-min walk test has important prognostic implications. In their study, patients who walked $>332 \mathrm{~m}$ had a 3 -yr survival of $\sim 90 \%$, whereas, for those who walked $<332 \mathrm{~m}$, the $3-\mathrm{yr}$ survival decreased to a mere $20 \%$. Among patients who received long-term treatment with intravenous epoprostenol, those who achieved a walking distance of $>380 \mathrm{~m}$ reached a 5 -yr survival of $70 \%$, whereas patients who covered lesser distances showed a 5-yr survival of $40 \%$ [5]. A recently published study of WeNSEL et al. [25] examined the prognostic importance of functional assessment, invasive haemodynamic studies and CPET. Clearly, $V^{\prime} \mathrm{O}_{2}$, max and peak systolic blood pressure during exercise, both determined by CPET, were the strongest independent predictors of survival. Patients with a $V^{\prime} \mathrm{O}_{2}$, max of $<10.4 \mathrm{~mL} \cdot \mathrm{kg}^{-1} \cdot \mathrm{min}^{-1}$ showed a 1 -yr survival of only $50 \%$, whereas patients with a $V^{\prime} \mathrm{O}_{2}$, max of $>10.4 \mathrm{~mL} \cdot \mathrm{kg}^{-1} \cdot \mathrm{min}^{-1}$ exhibited a $1-\mathrm{yr}$ survival of $90 \%$. Even more strikingly, patients with a peak systolic blood pressure of $<120 \mathrm{mmHg}$ during exercise showed a 1-yr survival of only
$34 \%$, whereas those with higher peak systolic blood pressures exhibited a 1-yr survival of $93 \%$.

Based on these data, the present authors believe that the increases in 6-min walking distance, $V^{\prime} \mathrm{O}_{2}$, max and peak systolic blood pressure seen with combination treatment in the current patients not only reflect improved exercise capacity but also are of significant prognostic importance. CPET has not yet been widely used to address the efficacy of medical treatment in patients with pulmonary hypertension. However, the observations of WENSEL et al. [25] suggest that it might be worthwhile to include CPET in the study protocols of further clinical trials.

The present study has several limitations, among which the uncontrolled design, small sample size and short observation period deserve special attention. Nevertheless, the present results demonstrate the feasibility of combination treatment and therefore open novel therapeutic perspectives for patients with PPH. Clearly, long-term studies comparing the effects of combination therapy with both prostanoids and endothelinreceptor antagonists alone are ultimately required to guide treatment. It is also important to point out that these first experiences with combination treatment were obtained solely in patients with $\mathrm{PPH}$ and that it remains to be shown whether combination treatment is also effective in other groups of PAH patients.

It is worthy of note that the present study provides no evidence that combination treatment is more effective than treatment with prostanoids or bosentan alone. In all of the current patients, prostanoids showed initial beneficial effects, as indicated by improvements in 6-min walk test results and haemodynamics. However, all patients eventually experienced progressive clinical deterioration and it is not known whether the prostanoids still had a therapeutic effect. It is possible that replacing prostanoids with bosentan might have been as effective as adding bosentan to the prostanoids. It is crucial to address this aspect in future studies before combination therapy is considered a standard treatment for pulmonary hypertension.

When the combination treatment was started, there were some safety concerns about the co-administration of prostanoids and bosentan. It was feared that hypotensive complications would arise in these patients, several of whom already showed blood pressure levels in the hypotensive range. However, no complications were observed that would have suggested significant hypotension. Moreover, as already mentioned above, the peak systolic blood pressure during exercise was significantly increased in the patients receiving combination treatment. As expected from previous reports on bosentan in patients with heart failure, there were some cases of fluid retention causing leg oedema that were mostly transient. Anaemia was not a problem in the present study population. Anticoagulation with the oral anticoagulant phenprocoumon was altered in the majority of patients, probably due to the induction by bosentan of cytochrome $\mathrm{P}_{450}$-dependent metabolism [26, 27]. Thus close monitoring of the INR is indispensable in patients undergoing anticoagulation therapy with phenprocoumon as long as they receive bosentan.

In conclusion, the present study has three novel findings. First, addition of the endothelin- receptor antagonist bosentan to the therapy of selected primary pulmonary hypertension patients already receiving beraprost or inhaled iloprost resulted in substantial improvement in 6-min walk test results. Secondly, cardiopulmonary exercise testing revealed that the addition of bosentan caused significant improvements in maximal oxygen consumption, anaerobic threshold, oxygen pulse, ventilatory efficacy and peak systolic blood pressure during exercise. Thirdly, at least throughout the relatively short observation period, combination of nonparenteral prostanoids and bosentan was safe. These findings 
offer a novel therapeutic perspective for primary pulmonary hypertension patients in whom treatment with nonparenteral prostanoids alone is not sufficiently effective and should prompt rigorous studies to determine whether combination treatment with prostanoids and bosentan is more effective than any of these substances alone.

\section{References}

1. Rubin LJ. Primary pulmonary hypertension. $N$ Engl J Med 1997; 336: 111-117.

2. D'Alonzo GE, Barst RJ, Ayres SM, et al. Survival in patients with primary pulmonary hypertension. Results from a national prospective registry. Ann Intern Med 1991; 115: 343-349.

3. Barst RJ, Rubin LJ, Long WA, et al. A comparison of continuous intravenous epoprostenol (prostacyclin) with conventional therapy for primary pulmonary hypertension. $N$ Engl J Med 1996; 334: 296-302.

4. McLaughlin VV, Genthner DE, Panella MM, Rich S. Reduction in pulmonary vascular resistance with long-term epoprostenol (prostacyclin) therapy in primary pulmonary hypertension. $N$ Engl J Med 1998; 338: 273-277.

5. Sitbon $\mathrm{O}$, Humbert $\mathrm{M}$, Nunes $\mathrm{H}$, et al. Long-term intravenous epoprostenol infusion in primary pulmonary hypertension. Prognostic factors and survival. J Am Coll Cardiol 2002; 40: 780-788.

6. McLaughlin VV, Shillington A, Rich S. Survival in primary pulmonary hypertension: the impact of epoprostenol therapy. Circulation 2002; 106: 1477-1482.

7. Simonneau G, Barst RJ, Galie N, et al. Continuous subcutaneous infusion of treprostinil, a prostacyclin analogue, in patients with pulmonary arterial hypertension. A double-blind, randomized, placebo-controlled trial. $\mathrm{Am}$ J Respir Crit Care Med 2002; 165: 800-804.

8. Galie N, Humbert M, Vachiery JL, et al. Effects of beraprost sodium, an oral prostacyclin analogue, in patients with pulmonary arterial hypertension: a randomized, doubleblind, placebo-controlled trial. J Am Coll Cardiol 2002; 39: 1496-1502.

9. Olschewski H, Simonneau G, Galie N, et al. Inhaled iloprost for severe pulmonary hypertension. N Engl J Med 2002; 347: 322-329.

10. Hoeper MM, Spiekerkoetter E, Westerkamp V, Gatzke R, Fabel H. Continuous intravenous iloprost for treatment failure of aerosolized iloprost in patients with pulmonary arterial hypertension. Eur Respir J 2002; 20: 339-343.

11. Lüscher TF, Barton M. Endothelins and endothelin receptor antagonists. Therapeutic considerations for a novel class of cardiovascular drugs. Circulation 2000; 102: 2434-2440.

12. Channick RN, Simonneau G, Sitbon O, et al. Effects of the dual endothelin-receptor antagonist bosentan in patients with pulmonary hypertension: a randomised placebo-controlled study. Lancet 2001; 358: 1119-1123.
13. Rubin LJ, Badesch DB, Barst RJ, et al. Bosentan therapy for pulmonary arterial hypertension. $N$ Engl J Med 2002; 346: 896-903.

14. Ueno M, Miyauchi T, Sakai S, Yamauchi-Kohno R, Goto K, Yamaguchi I. A combination of oral endothelin-A receptor antagonist and oral prostacyclin analogue is superior to each drug alone in ameliorating pulmonary hypertension in rats. J Am Coll Cardiol 2002; 40: 175-181.

15. Hoeper MM, Olschewski H, Ghofrani HA, et al. A comparison of the acute hemodynamic effects of inhaled nitric oxide and aerosolized iloprost in primary pulmonary hypertension. J Am Coll Cardiol 2000; 35: 176-182.

16. Hoeper MM, Schwarze M, Ehlerding S, et al. Long-term treatment of primary pulmonary hypertension with aerosolized iloprost, a prostacyclin analogue. $N$ Engl J Med 2000; 342: $1866-1870$

17. Gessler T, Schmehl T, Hoeper MM, et al. Ultrasonic versus jet nebulization of iloprost in severe pulmonary hypertension. Eur Respir J 2001; 17: 14-19.

18. Guyatt GH, Sullivan MJ, Thompson PJ, et al. The 6-minute walk: a new measure of exercise capacity in patients with chronic heart failure. Can Med Assoc J 1985; 132: 919-923.

19. Sun XG, Hansen JE, Oudiz RJ, Wasserman K. Exercise pathophysiology in patients with primary pulmonary hypertension. Circulation 2001; 104: 429-435.

20. Sun XG, Hansen JE, Oudiz RJ, Wasserman K. Gas exchange detection of exercise-induced right-to-left shunt in patients with primary pulmonary hypertension. Circulation 2002; 105: 54-60.

21. Wasserman K, Hansen JE, Sue DY. Principles of Exercise Testing and Interpretation. Baltimore, MD, Lippincott Williams \& Wilkens, 1999.

22. Wensel R, Opitz CF, Ewert R, Bruch L, Kleber FX. Effects of iloprost inhalation on exercise capacity and ventilatory efficiency in patients with primary pulmonary hypertension. Circulation 2000; 101: 2388-2392.

23. Blumberg FC, Riegger GA, Pfeifer M. Hemodynamic effects of aerosolized iloprost in pulmonary hypertension at rest and during exercise. Chest 2002; 121: 1566-1571.

24. Miyamoto S, Nagaya N, Satoh T, et al. Clinical correlates and prognostic significance of six-minute walk test in patients with primary pulmonary hypertension. Comparison with cardiopulmonary exercise testing. Am J Respir Crit Care Med 2000; 161: 487-492.

25. Wensel R, Opitz C, Anker SD, et al. Assessment of survival in patients with primary pulmonary hypertension: importance of cardiopulmonary exercise testing. Circulation 2002; 106: 319-324.

26. Weber C, Banken L, Birnboeck H, Schulz R. Effect of the endothelin-receptor antagonist bosentan on the pharmacokinetics and pharmacodynamics of warfarin. J Clin Pharmacol 1999; 39: 847-854

27. Harder S, Thurmann P. Clinically important drug interactions with anticoagulants. An update. Clin Pharmacokinet 1996; 30: 416-444. 